\title{
Experimental investigation of transverse mixing in porous media under helical flow conditions
}

Ye, Yu; Chiogna, Gabriele; Cirpka, Olaf A.; Grathwohl, Peter; Rolle, Massimo

Published in:

Physical Review E

Link to article, DOI:

10.1103/PhysRevE.94.013113

Publication date:

2016

Document Version

Publisher's PDF, also known as Version of record

Link back to DTU Orbit

Citation (APA):

Ye, Y., Chiogna, G., Cirpka, O. A., Grathwohl, P., \& Rolle, M. (2016). Experimental investigation of transverse mixing in porous media under helical flow conditions. Physical Review E, 94(1), [013113].

https://doi.org/10.1103/PhysRevE.94.013113

\section{General rights}

Copyright and moral rights for the publications made accessible in the public portal are retained by the authors and/or other copyright owners and it is a condition of accessing publications that users recognise and abide by the legal requirements associated with these rights.

- Users may download and print one copy of any publication from the public portal for the purpose of private study or research.

- You may not further distribute the material or use it for any profit-making activity or commercial gain

- You may freely distribute the URL identifying the publication in the public portal 


\title{
Experimental investigation of transverse mixing in porous media under helical flow conditions
}

\author{
Yu Ye, ${ }^{1,2}$ Gabriele Chiogna, ${ }^{1,3}$ Olaf A. Cirpka, ${ }^{1}$ Peter Grathwohl, ${ }^{1}$ and Massimo Rolle ${ }^{1,4, *}$ \\ ${ }^{1}$ Center for Applied Geoscience, University of Tübingen, Hölderlinstraße 12, D-72074 Tübingen, Germany \\ ${ }^{2}$ State Key Laboratory of Hydrology-Water Resources and Hydraulic Engineering, Hohai University, Nanjing, China \\ ${ }^{3}$ Faculty of Civil, Geo and Environmental Engineering, Technical University of Munich, Arcistraße 21, D-80333 Munich, Germany \\ ${ }^{4}$ Department of Environmental Engineering, Technical University of Denmark, Miljøvej Building 115, DK-2800 Lyngby, Denmark
}

(Received 31 March 2016; revised manuscript received 6 July 2016; published 22 July 2016)

\begin{abstract}
Plume dilution and transverse mixing can be considerably enhanced by helical flow occurring in threedimensional heterogeneous anisotropic porous media. In this study, we perform tracer experiments in a fully threedimensional flow-through chamber to investigate the effects of helical flow on plume spiraling and deformation, as well as on its dilution. Porous media were packed in angled stripes of materials with different grain sizes to create blocks with macroscopically anisotropic hydraulic conductivity, which caused helical flows. Steady-state transport experiments were carried out by continuously injecting dye tracers at different inlet ports. High-resolution measurements of concentration and flow rates were performed at 49 outlet ports. These measurements allowed quantifying the spreading and dilution of the solute plumes at the outlet cross section. Direct evidence of plume spiraling and visual proof of helical flow was obtained by freezing and slicing the porous media at different cross sections and observing the dye-tracer distribution. We simulated flow and transport to interpret our experimental observations and investigate the effects of helical flow on mixing-controlled reactive transport. The simulation results were evaluated using metrics of reactive mixing such as the critical dilution index and the length of continuously injected steady-state plumes. The results show considerable reaction enhancement, quantified by the remarkable decrease of reactive plume lengths (up to four times) in helical flows compared to analogous scenarios in uniform flows.
\end{abstract}

DOI: 10.1103/PhysRevE.94.013113

\section{INTRODUCTION}

Mixing of reactants is fundamental for the occurrence of chemical and biological reactions both in natural and in engineered flows [1-4]. In turbulent flows, such as in many examples of atmospheric and surface-water flows as well as in engineered systems like mixers, mixing is comparably strong and reaction rates are often controlled by reaction kinetics [5]. By contrast, in creeping flows occurring in porous media such as aquifers, oil reservoirs, and packed bed reactors, slow diffusive processes determine the rate of mixing and, hence, reactions may be controlled by insufficient mixing [6,7]. Microfluidics experiments [8-11] and pore-scale simulations [6,12-17] have emphasized the controlling role of pore-scale physics on conservative and reactive transport. The effects of pore-scale processes do not vanish and affect solute transport and mixing in porous media also at larger scales $[18,19]$. Physical heterogeneity of porous formations is a key feature that has been recognized to control the extent of mixing in geologic media. In such systems, the interplay between mixing and reactive processes has been extensively investigated by means of numerical simulations and theoretical analysis [20-25]. A feature of porous media that has received considerably less attention than heterogeneity in the study of mixing processes is anisotropy. Anisotropic structures can result in flow fields with complex topology, nonzero helicity density, and entailing whirling streamlines [26-28]. Modeling studies have started to address the link between the complexity of steady-state flows in three-dimensional anisotropic domains and the

*Corresponding author: masro@env.dtu.dk deformation, stretching, folding of solute plumes [29-31] and, ultimately, plume dilution and mixing [32].

The current study aims at extending and deepening the findings presented in a recent Letter [33], which showed first experimental evidence of the occurrence of helical flow in porous media. We present results of laboratory flow-through experiments providing visual proof of helical flow in heterogeneous anisotropic porous media. Color-tracer distributions of steady-state plumes examined in different cross sections of the domain show the effects of whirling streamlines and their impact on plume deformation. We also investigate the influence of grain sizes and the effects of different tracer-injection positions on plume dilution. Finally, we use a numerical flow and transport model, validated with the results of the conservative-tracer laboratory experiments, to explore the effects of helical flow on reactive transport. We consider a mixing-controlled reactive transport scenario in which a continuously injected reactant plume undergoes an instantaneous complete bimolecular reaction. The critical dilution index, the effective dispersion coefficients, and the length of reactive plumes are the metrics selected to quantify the reaction enhancement due to helical flow.

\section{MATERIAL AND METHODS}

\section{A. Flow-through experiments}

Laboratory flow-through experiments were carried out in a three-dimensional flow-through chamber with inner dimensions of $30 \mathrm{~cm} \times 7.7 \mathrm{~cm} \times 10 \mathrm{~cm}$ [Fig. 1(a)]. The chamber is made of acrylic glass and has 25 equally spaced $(1.54 \mathrm{~cm}$ spacing) injection ports at the inlet and 49 equally spaced (1.1 cm spacing) extraction ports at the outlet. Syringe needles 

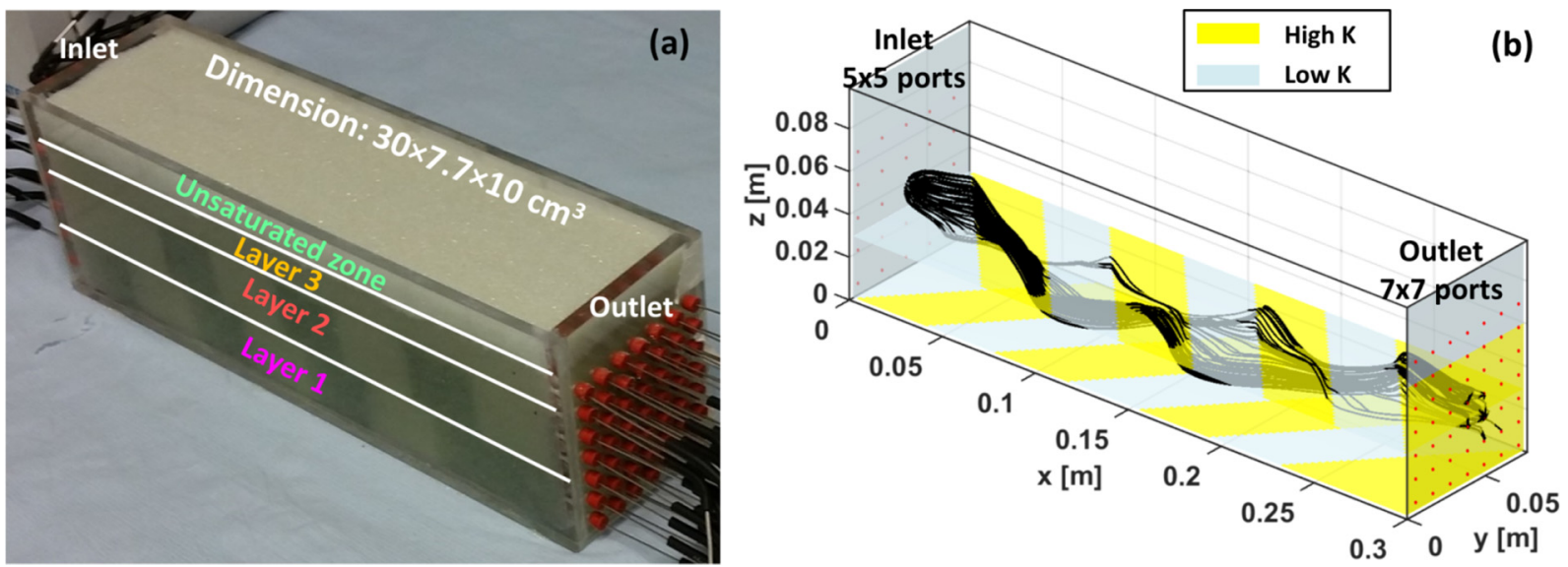

FIG. 1. (a) Photograph of the experimental setup with marked layers. (b) Inner heterogeneous anisotropic structure of the porous medium, built with high and low hydraulic conductivity angled stripes, and streamlines traced from the central injection port.

piercing rubber septa were used to inject and extract water at the inlet and outlet ports. High-precision peristaltic pumps with 24 channels (Ismatec, Glattbrugg, Switzerland) were used to establish steady-state flow conditions during the experimental runs. Two sets of experiments were carried out using different types of glass beads (Sigmund Lindner, Warmensteinach, Germany) arranged to create heterogeneous anisotropic porous media in the flow-through chamber. In the first series of experiments, fine materials with grain sizes of $0.4-0.6 \mathrm{~mm}$ (indicated as FF) and 1.5-2.0 mm (indicated as FC) were used for the construction of low and high conductivity stripes, respectively. In the second series of experiments, coarser materials with the grain sizes of $0.6-0.9 \mathrm{~mm}$ (indicated as $\mathrm{CF}$ ) and $2.4-2.9 \mathrm{~mm}$ (indicated as CC) were used to pack the heterogeneous anisotropic medium. The ratio of the average grain size between fine and coarse materials in the two setups is identical (i.e., 3.5). In each experiment, the two types of glass beads were packed in alternating angled stripes in two distinct layers [layer 1 and layer 2 in Fig. 1(a)] to achieve a simplified representation of herringbone cross-stratification, observed in subsurface sedimentary depositional environments [34]. In layer 3, finer glass beads (FF or $\mathrm{CF}$ ) were homogeneously packed. Such a layer is necessary to facilitate maintaining water-saturated conditions in the coarser porous medium in layers 1 and 2. The thickness of layers 1 and 2 was 3.08 $\mathrm{cm}$, whereas layer 3 had a thickness of $1.54 \mathrm{~cm}$. We consider unconfined conditions in this work, that is, no sealing at the top of the filling was applied. To prevent capillary rise to the surface, dry coarse glass beads were placed on top of layer 3 , maintaining an unsaturated zone. The porosity of the porous media was determined gravimetrically and a value of 0.4 was found for all types of glass beads used in the experiments. The hydraulic conductivity was calculated according to the grain size [35]. The porous media built for the flow-through experiments have a spatially heterogeneous and macroscopically anisotropic hydraulic conductivity distribution, which is a key feature to generate helical flow.

All experiments were performed at an average flow velocity of $3 \mathrm{~m} /$ day. After establishing steady-state flow conditions, a tracer solution of sodium fluorescein (CAS 518-47-8) with a concentration of $15 \mathrm{mg} / \mathrm{l}$ was injected through a single injection port, while tracer-free water was injected through all other ports. To ensure the achievement of steady-state transport conditions, we flushed eight pore volumes before starting sampling. Samples were collected at the $49(7 \times 7$ array $)$ extraction ports at the outlet and the concentrations of the collected samples were measured using a UV fluorescence spectrometer (Perkin Elmer LS-3B). The flow rate at each extraction port was determined by weighting the collected samples at specific time intervals.

A second color tracer (New Coccine, CAS 2611-82-7) was used in an experiment aimed at visualizing the cross-sectional concentration distributions at different distances within the flow-through system. This experiment was conducted in the porous medium made of FF and FC glass beads, and New Coccine was selected as tracer since its red color was easier to distinguish from the color of the glass beads compared to fluorescein. To enable visual inspection of the tracer plume at different cross sections, the porous medium was frozen at $-20^{\circ} \mathrm{C}$ and sliced at distances of $11,15,24$, and $29 \mathrm{~cm}$ from the inlet. Photographs of the different cross sections were taken to visualize the spatial distribution of the color-tracer concentration.

\section{B. Flow and transport modeling}

Flow and transport simulations were performed to (i) interpret the experimental observations; (ii) compute metrics of plume spreading and dilution and compare the model results with the experimental findings; and (iii) extend the analysis to study the impact of helical flow on mixing-controlled reactive transport.

We used the scheme proposed by [32] to solve flow and transport in three-dimensional anisotropic porous media with the same geometry, boundary conditions, as well as arrangement of hydraulic conductivity zones as in the experimental setup.

Steady-state flow was calculated according to the continuity equation and Darcy's law:

$$
\nabla \cdot[\mathbf{q}(\mathbf{x})]=\nabla \cdot[-\mathbf{K}(\mathbf{x}) \nabla \phi(\mathbf{x})]=0,
$$



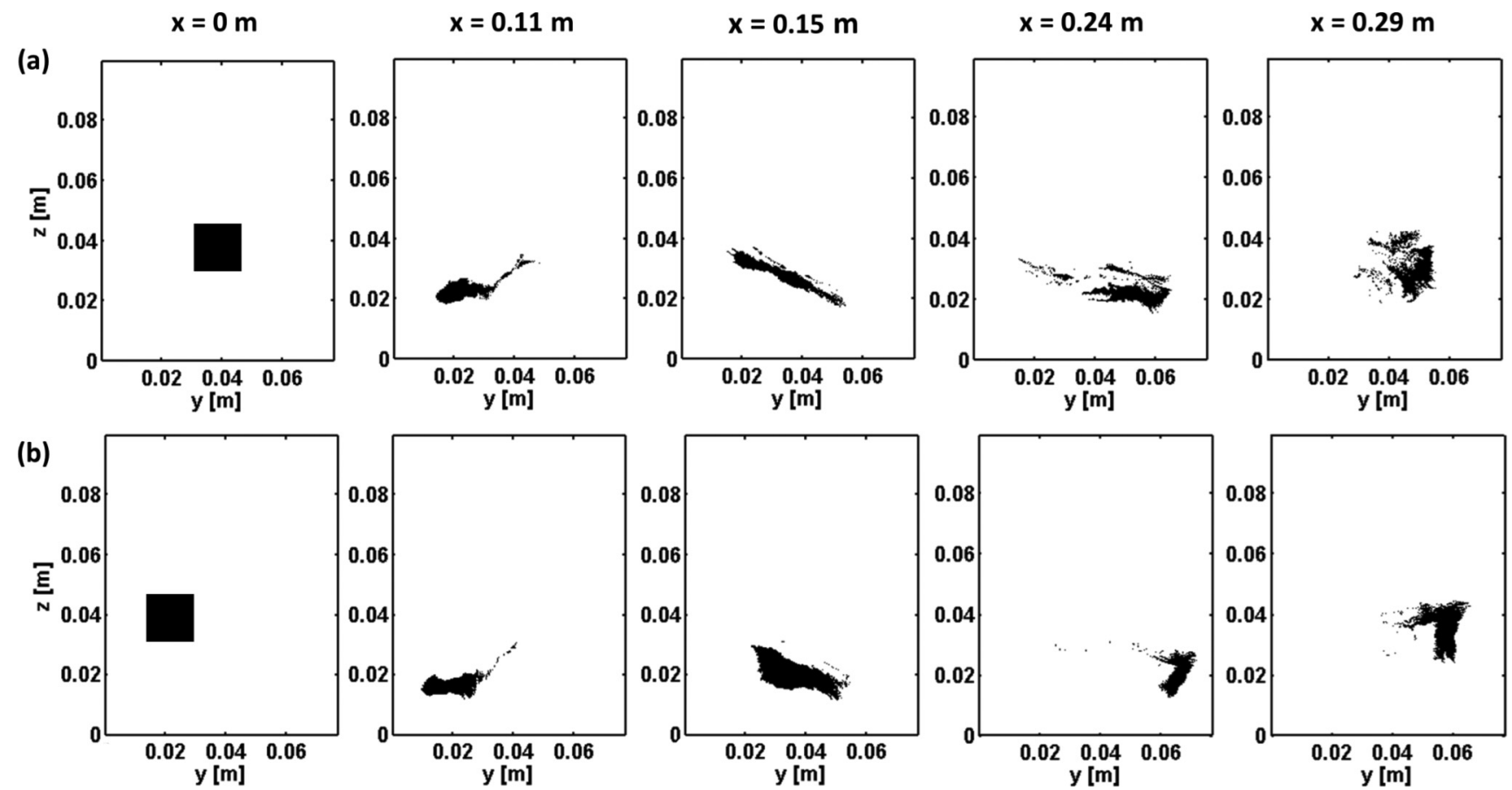

FIG. 2. Effect of the complex flow field on plume deformation at different longitudinal distances of the domain. Simulated patterns of streamlines injected from (a) a centered injection and (b) a noncentered injection, corresponding to the experiment using New Coccine as tracer (Fig. 3).

where $\mathbf{q}\left[L T^{-1}\right]$ denotes the specific discharge vector, $\mathbf{x}[L]$ is the vector of spatial coordinates, $\mathbf{K}\left[L T^{-1}\right]$ is the hydraulic conductivity tensor (here considered locally isotropic as the stripes of different materials are spatially resolved), and $\phi[L]$ is the hydraulic head. Equation (1) was solved considering constant-flux boundary conditions at the injection and extraction ports and no-flow boundary conditions at the other sides of the flow-through chamber. The complex flow field within the heterogeneous anisotropic porous medium is shown by the streamlines traced from the central injection port to the outlet [Fig. 1(b)], using a particle tracking scheme based on Pollock's semianalytical method [36]. The computed streamlines are helical curves in the three-dimensional porous medium, twisting along the travel distance. Streamlines originating from the same injection port may evolve quite differently inside the porous medium, thus causing considerable deformation of an injected solute plume. Such effects can be visualized by plotting how the cross section of a plume in the $y-z$ planes is deformed as a function of distance $x$. We do this by tracking 2500 streamlines originating from a given source zone at the inlet. Figure 2(a) illustrates the effects of the complex flow field on the plume shape at selected cross sections for a centered and symmetric injection zone (i.e., central inlet port in the experimental setup). The injected plume is displaced, stretched, and squeezed according to the pattern of the streamlines injected through the source zone. It is evident that diverging streamlines can even cause the splitting of the plume. Figure 2(b) shows the case where the plume is injected at a noncentral inlet port corresponding to the experiment using New Coccine as tracer. The experimental results and the transport simulations will be discussed in the next sections; however, the visualization of the streamlines patterns at different cross sections is illustrative to isolate the impact of the complex helical flow on the dynamics of plume deformation (i.e., purely advective effects, without diffusive/dispersive mass transfer processes).

The governing equation for the conservative transport problem is the steady-state advection-dispersion equation:

$$
\mathbf{v} \cdot \nabla c-\nabla \cdot(\mathbf{D} \nabla c)=0
$$

where $\mathbf{v}=\mathbf{q} / \theta\left[L T^{-1}\right]$ is the average linear velocity vector, $\theta[-]$ is the porosity, $c\left[M L^{-3}\right]$ is the concentration, and $\mathbf{D}$ $\left[L^{2} T^{-1}\right]$ is the hydrodynamic dispersion tensor.

The transport equation [Eq. (2)] was solved considering constant concentrations at the inlet boundary $(15 \mathrm{mg} / 1$ for the tracer-injection port and $0 \mathrm{mg} / \mathrm{l}$ for the other ports where clean water was injected). For continuously injected plumes, under steady-state conditions, the key dispersion term is the one in the transverse direction, whereas longitudinal dispersion can be neglected [37]. It is therefore important to provide an accurate description of the transverse dispersion coefficient. We considered the nonlinear compound-specific parameterization of [38]:

$$
D_{t}=D_{p}+D_{\mathrm{aq}}\left(\frac{P e^{2}}{P e+2+4 \delta^{2}}\right)^{\beta}
$$

taking into account the dependence of mechanical dispersion on the aqueous diffusivity of the transported solute, which stems from incomplete mixing at the pore scale [39-41]. Here $D_{p} \approx D_{\mathrm{aq}} \theta\left[L^{2} T^{-1}\right]$ denotes the pore diffusion coefficient, $D_{\text {aq }}\left[L^{2} T^{-1}\right]$ is the aqueous diffusion coefficient, $P e=$ $v d / D_{\text {aq }}[-]$ is the grain Péclet number, $v\left[L T^{-1}\right]$ is the absolute value of the velocity vector $\mathbf{v}, d[L]$ is the average grain size, $\delta[-]$ is the ratio between the length of a pore 
channel and its hydraulic radius, and $\beta[-]$ is an empirical exponent that accounts for the degree of incomplete mixing within the pore channels. The parameters reported by [42] (i.e., $\beta=0.5$ and $\delta=5.37$ ) were used in this study.

\section{METRICS OF CONSERVATIVE AND REACTIVE MIXING}

\section{A. Quantification of plume spreading and dilution}

The metrics selected to evaluate the outcomes of the conservative-tracer experiments are the low-order moments of the concentration distribution and the flux-related dilution index.

Second central spatial moments are useful to characterize the spreading of solute plumes. For the steady-state tracer experiments with continuous injection, the moments in the transverse horizontal and vertical directions are informative of the concentration distribution at a given cross section and were calculated at the outlet of the flow-through setup:

$$
\begin{aligned}
M_{2 C, h} & =\frac{\int_{0}^{w_{\text {tot }}}\left(y-M_{1, h}\right)^{2} c_{h} d y}{\int_{0}^{w_{\text {tot }}} c_{h} d y}, \\
M_{2 C, v} & =\frac{\int_{0}^{h_{\text {tot }}\left(z-M_{1, v}\right)^{2} c_{v} d z}}{\int_{0}^{h_{\text {tot }}} c_{v} d z},
\end{aligned}
$$

where $w_{\text {tot }}[L]$ and $h_{\text {tot }}[L]$ are the total width and height of the three-dimensional domain, $M_{1, h}[L]$ and $M_{1, v}[L]$ are the first spatial moment in horizontal and vertical directions, defined as:

$$
\begin{aligned}
& M_{1, h}=\frac{\int_{0}^{w_{\text {tot }}} y c_{h} d y}{\int_{0}^{w_{\text {tot }}} c_{h} d y}, \\
& M_{1, v}=\frac{\int_{0}^{h_{\text {tot }}} z c_{v} d z}{\int_{0}^{h_{\text {tot }}} c_{v} d z},
\end{aligned}
$$

and $c_{h}=\int_{0}^{h_{\text {tot }}} c d z / h_{\text {tot }}$ and $c_{v}=\int_{0}^{w_{\text {tot }}} c d y / w_{\text {tot }}$ represent the average concentration along the vertical and horizontal directions, respectively.

The flux-related dilution index, $E_{Q}\left[L^{3} T^{-1}\right]$, is a useful metric to quantify plume dilution and is defined as [43]:

$E_{Q}(x)=\exp \left\{-\int_{\Omega}\left[p_{Q}(x, y, z) \ln p_{Q}(x, y, z)\right] q_{x}(x, y, z) d A\right\}$,

where $\Omega$ is the cross section perpendicular to the longitudinal direction $x, p_{Q}=c / \int_{\Omega} c q_{x} d A\left[T L^{-3}\right]$ is the flux-weighted probability density function of the solute mass, and $q_{x}\left[L T^{-1}\right]$ is the specific discharge in the main flow direction. The flux-related dilution index is a metric based on the Shannon entropy and was derived following the volumetric dilution index $E\left[L^{3}\right]$ [44], which was proposed to capture the increase of volume of fluid occupied by the solute during transient transport of a solute cloud. $E_{Q}$ is a volumetric flow rate and quantifies the distribution of the solute mass flux over the water flux along the travel distance. Even though the logarithm of a dimensional property $\left(p_{Q}\right)$ appears in Eq. (8), the equation is dimensionally correct and represents the limit of a discrete exponential of the plume's entropy in which the arguments of exponents and logarithms are dimensionless [44]. In our experiments, we determined the flux-related dilution index in a discrete form using the concentrations and flow rates measured at the inlet and the outlet ports. The difference between the outlet and inlet values of the flux-related dilution index directly quantifies the extent of plume dilution due to lateral mass exchange in the flow-through setup.

\section{B. Quantification of reactive mixing}

Numerical simulations are used to investigate the effects of helical flow on reactive transport. We consider the same flow-through domain used in the experiments and a simple mixing-controlled instantaneous bimolecular reaction of the form $A+B \rightarrow C$. Species $A$ is the plume injected in one port, while species $B$ is the reactant present in the ambient water and introduced in all other ports. Assuming the same aqueous diffusion coefficient for both reactants, a virtual conservative compound $X[-]$ (denoted as mixing ratio) can be considered to model the reactive transport problem $[45,46]$. The mixing ratio is defined as the volumetric fraction of the injected solution in the mixture and can be used to map the concentration of reactants and products of an instantaneous bimolecular reaction according to the procedure described by Cirpka and Valocchi [46]. In our study, the concentration of $X$ is unity at the injection port of $A$ and it is 0 in the ambient water. We define the critical mixing ratio according to [46] as $X_{\text {crit }}=c_{B}^{\mathrm{amb}} /\left(c_{B}^{\mathrm{amb}}+c_{A}^{\mathrm{in}}\right)$, where $c_{B}^{\mathrm{amb}}$ is the concentration of species $B$ in the inlet ambient water and $c_{A}^{\mathrm{in}}$ is the concentration of species $A$ at the central injection port through which the reactive plume is injected in the flow-through domain. At locations where $X=X_{\text {crit }}$, the concentrations of both reactants are zero.

In uniform three-dimensional, laterally unbounded flow, the mixing ratio satisfies the analytical solution for conservative transport of solute plumes continuously injected by a square source [47]:

$$
\begin{aligned}
X(x, y, z)= & \frac{1}{4}\left\{\operatorname{erf}\left[\frac{y+Y / 2}{2 \sqrt{D_{t} x / v}}\right]-\operatorname{erf}\left[\frac{y-Y / 2}{2 \sqrt{D_{t} x / v}}\right]\right\} \\
& \times\left\{\operatorname{erf}\left[\frac{z+Z / 2}{2 \sqrt{D_{t} x / v}}\right]-\operatorname{erf}\left[\frac{z-Z / 2}{2 \sqrt{D_{t} x / v}}\right]\right\},
\end{aligned}
$$

where $\operatorname{erf}(\cdot)$ is the error function, and $Y[L]$ and $Z[L]$ denote the source width and the source thickness, which are identical (i.e., $1.54 \mathrm{~cm}$ ) in our study. The end of the plume is defined as the point where species $A$ is completely consumed by the reaction, i.e., where the mixing ratio equals $X_{\text {crit }}$. Thus, the plume length, $L[L]$, is defined as the minimal distance from the source at which $X \leqslant X_{\text {crit }}$ within the entire cross section orthogonal to the average flow direction. The length of a reactive plume is a useful metric to quantify the effects of mixing on reactive transport. For a three-dimensional homogeneous isotropic domain, the plume length of reactant $A$ can be derived from Eq. (9) following the procedure outlined in Appendix A and reads as:

$$
L=\frac{v Y^{2}}{16 D_{t}\left[\operatorname{erf}^{-1}\left(\sqrt{X_{\text {crit }}}\right)\right]^{2}},
$$


where $\operatorname{erf}^{-1}(\cdot)$ is the inverse error function. No analytical solution exists for the concentration distribution in the case of helical flow in heterogeneous anisotropic porous media. However, for transport in such domains we can define an effective dispersion coefficient, considering a homogeneous isotropic equivalent system with the same solute mass flux at the inlet and the same plume length observed in the heterogeneous anisotropic case. Therefore, $D_{t, \text { eff }}\left[L^{2} T^{-1}\right]$ is a useful metric of mixing enhancement in reactive transport and can be obtained from Eq. (10) as:

$$
D_{t, \mathrm{eff}}=\frac{v Y^{2}}{16 L\left[\operatorname{erf}^{-1}\left(\sqrt{X_{\mathrm{crit}}}\right)\right]^{2}} .
$$

Considering the effect of heterogeneity and anisotropy of the porous media on mixing, $D_{t, \text { eff }}$ is larger than the local transverse dispersion coefficient.

Finally, as the metric of reactive transport, we also consider the critical dilution index (CDI). This measure was introduced for the analysis of mixing-controlled reactions in two-dimensional porous media [48] and quantifies the amount of mixing required for the complete degradation of a reactive plume undergoing an instantaneous complete bimolecular reaction. The value of the critical dilution index can be computed from the flux-related dilution index of a conservative-tracer plume at the distance $L$, where $L$ is the length of the reactive plume considering the same flow field and the same mass flux as in the case of conservative transport. For three-dimensional transport, the critical dilution index and its first-order approximation are described in Appendix B. The analytical expression reads as:

$$
\mathrm{CDI}=E_{Q}(L)=\frac{E_{Q}(0)}{X_{\text {crit }}} \exp (1) .
$$

\section{RESULTS AND DISCUSSION}

\section{A. Conservative transport}

Figure 3 refers to the flow-through experiment conducted using New Coccine as color tracer and shows the concentration distribution at different cross sections after slicing the frozen porous medium containing the three-dimensional (3D) steadystate tracer plume. The operations of slicing and freezing, as well as the impossibility to stop diffusion, caused some disturbance of the color dye plume. Therefore, the results for the New Coccine plume should be interpreted only qualitatively. The upper plots [Fig. 3(a)] are photographs showing the location and spatial distribution of the tracer plume in cross sections at different longitudinal distances. For visual comparison, Fig. 3(a) also shows contour lines of simulated tracer concentrations. The same distributions are shown as color maps in Fig. 3(b). The simulated concentration values are normalized by the concentration at the source. The simulated concentrations qualitatively agree with the experimental results, demonstrating the capability of the model to predict the main features of flow and transport in the heterogeneous anisotropic domain and to reproduce the plume location, shape, and concentration distribution observed in the experiments. Both the experimental and modeling results show the importance of local transverse dispersion. In comparison

(a)
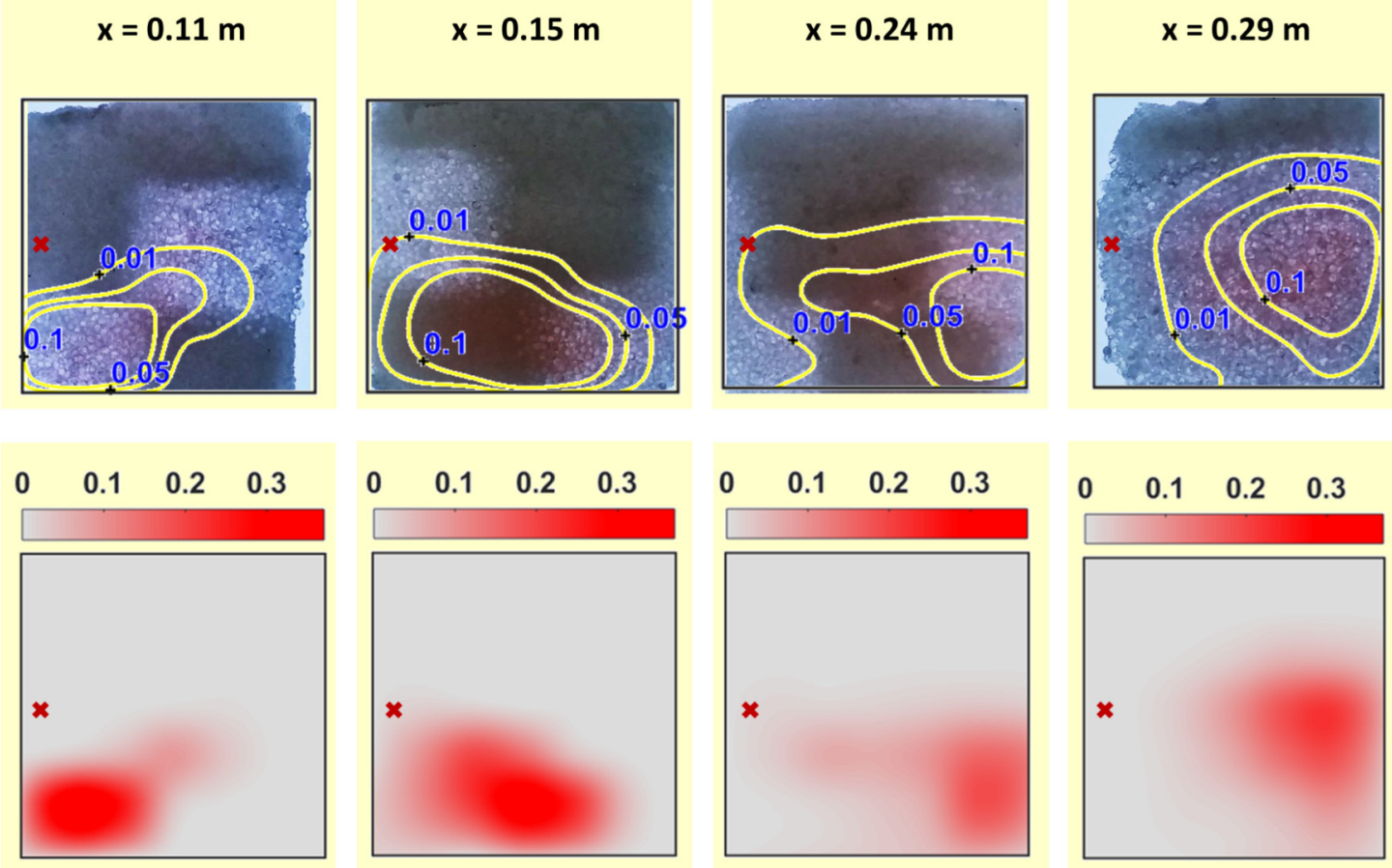

* Tracer injection point at the inlet

FIG. 3. Plume distribution at different cross sections. (a) Enhanced photographs showing the location and distribution of the New Coccine plume. Contour lines of simulated normalized concentrations are added to the photographs for direct comparison. (b) Concentration maps at the given longitudinal distances computed with the numerical model. 
to the simulated purely advective plume deformation shown in Fig. 2(b), the concentration distributions affected by transverse dispersion are much smoother and exhibit fewer fine-scale features. The flow field in our experimental setup causes the plume position to deviate from the tracer-injection points (red crosses), and leads to the deformation of the material surface of the plume. Lateral mass exchange by transverse hydrodynamic dispersion attenuates sharp concentration gradients and significantly affects the concentration distribution.

The experiment conducted using New Coccine as dye tracer aimed at visualizing the complex distribution and deformation of the steady-state plume inside the 3D flow-through setup. A series of experiments using fluorescein were performed in the fine (using FF and FC grain sizes to build the angled stripes with different hydraulic conductivity) and the coarse ( $\mathrm{CF}$ and $\mathrm{CC}$ grain sizes) heterogeneous anisotropic porous media, to quantitatively estimate the effect of the flow field on mixing of a conservative tracer. Normalized concentration distributions of fluorescein at the outlet of the flow-through system were measured at high resolution at the 49 outlet ports and interpolated using two-dimensional monotonicity-preserving cubic interpolation as implemented in MATLAB. These concentration measurements, together with the measurement of the flow rates at the different channels, provided the basis for the quantitative evaluation of transverse plume spreading and dilution in the flow-through setup. Figure 4 shows the experimental and numerical results of two experiments performed in the fine (FF and FC) and coarse (CF and $\mathrm{CC}$ ) porous media. In these experiments, the central inlet port was selected as the tracer-injection port (red crosses in Fig. 4). Maps of the experimental and simulated concentration distributions at the outlet are shown in Figs. 4(a) and 4(b) for the experiment in the fine porous medium and in Figs. 4(d) and 4(c) for the experiment carried out in the coarse porous medium. In both cases the measured and simulated results show good agreement concerning the location and the spatial distribution of the tracer as well as its peak concentration. Comparing the two experiments, the peak concentration of the plume at the outlet is higher for the setup packed with fine materials, indicating less transverse mixing in comparison to the case packed with coarse materials. Yet the position and the shape of the two plumes are similar. This confirms that the absolute values of the two grain sizes influence the degree of plume dilution (as expected from the parameterization of the transverse dispersion coefficient and from the dependence of the flux-related dilution index on transverse dispersion), but they do not affect the structure of the flow field and hence the shape of the steady-state plume if the ratio of grain sizes and thus the anisotropy ratio of the upscaled hydraulic conductivity are identical. Figures 3(c) and 3(f) compare the concentrations at the outlet ports between experimental and numerical results for the two setups. The experimental error $\varepsilon[-]$ was estimated using the reduced $\chi^{2}$ test [49], i.e., $\chi^{2}=\left(1 / n_{\text {ports }}\right) \sum_{i=1}^{n_{\text {ports }}}\left[\left(c_{i}^{\text {meas }}-c_{i}^{\text {sim }}\right)^{2} / \varepsilon^{2}\right]$, such that $\chi^{2}$ equals to unity. Here $n_{\text {ports }}[-]$ denotes the number of outlet ports (49 in our setup), $c_{i}^{\text {meas }}[-]$ is the measured normalized concentration at port $i$, and $c_{i}^{\text {sim }}[-]$ is the corresponding simulated value. The normalized error is plotted as dashed lines in Figs. 4(c) and 4(f). The error is smaller for the experiments packed with fine materials (grain size of $0.4-0.6 \mathrm{~mm}$ and $1.5-2.0 \mathrm{~mm}$ ) than for the case with coarse materials (grain size of $0.6-0.9 \mathrm{~mm}$ and $2.4-2.9 \mathrm{~mm}$ ). This may be related
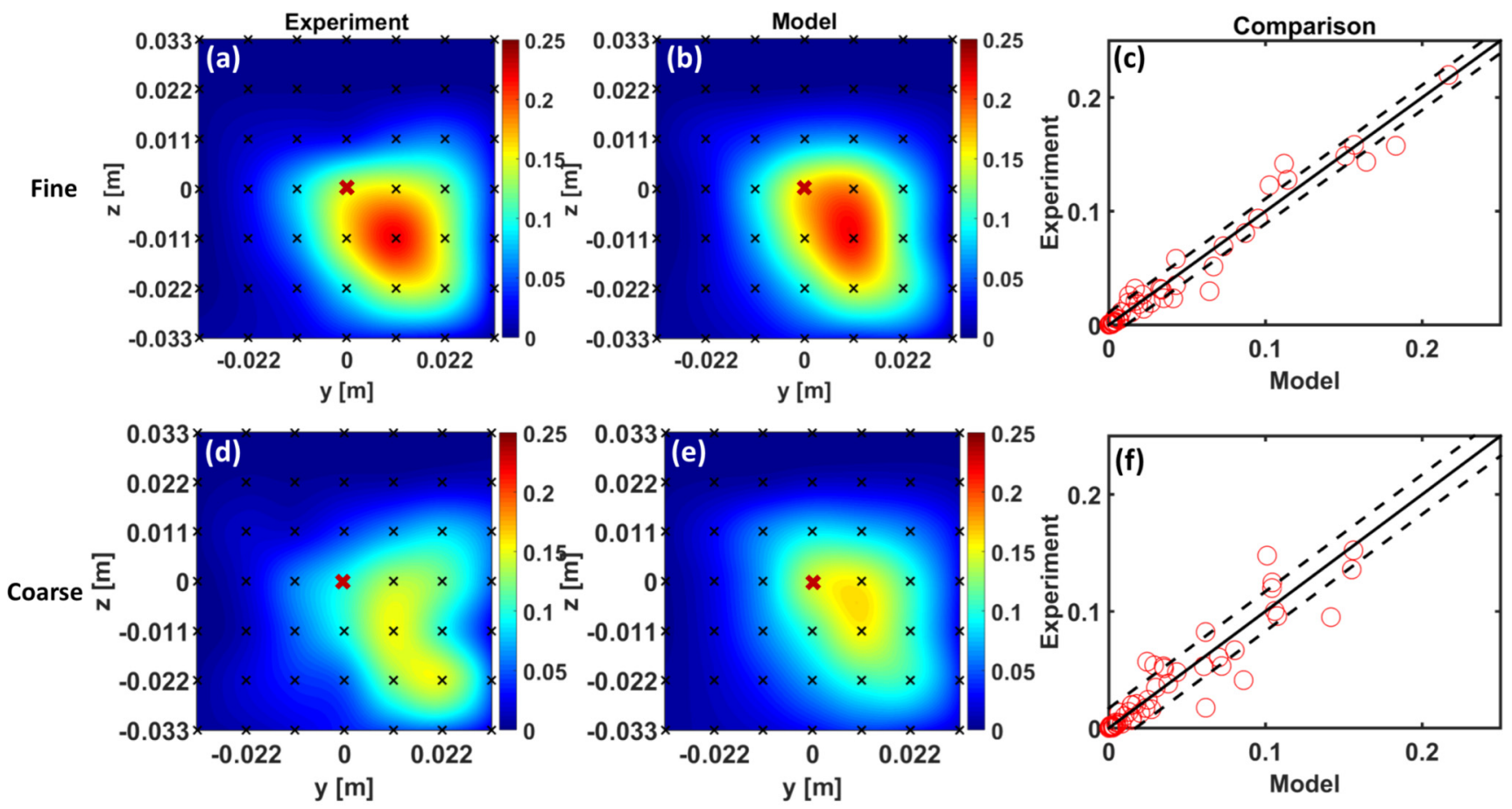

* Tracer injection point at the inlet

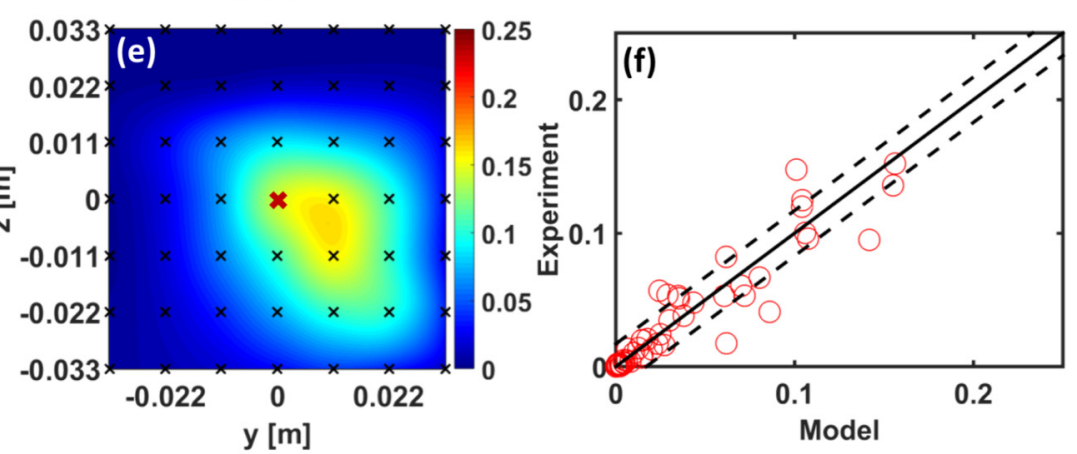

* Measuring point at the outlet

FIG. 4. Experimental and modeling results showing the normalized concentration distributions at the outlet of the heterogeneous anisotropic porous media packed with fine (grain size of $0.4-0.6 \mathrm{~mm}$ and $1.5-2.0 \mathrm{~mm}$ ) and coarse (grain size of $0.6-0.9 \mathrm{~mm}$ and 2.4-2.9 mm) materials. 

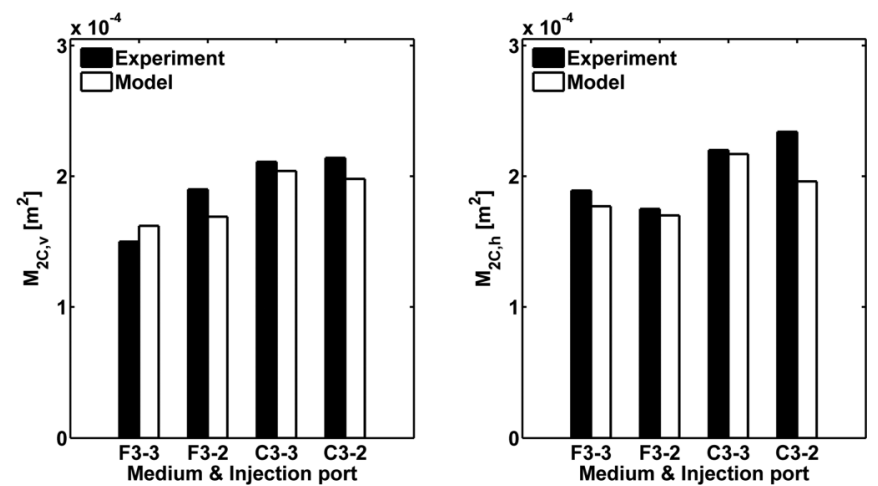

FIG. 5. Experimental and model results of the plume's second central moments in the vertical and horizontal transverse directions at the outlet of the flow-through setup packed with fine (F) and coarse (C) media and using the central inlet port ( $3-3$; Fig. 4$)$ and the neighboring one (3-2) as the tracer-injection ports.

to the accuracy in the construction of the angled stripes in the two setups. In fact, we observed that the grain size $\mathrm{CF}$ can intrude into $\mathrm{CC}$ better than FF into $\mathrm{FC}$, thus creating more irregular boundaries between the different stripes for the coarse porous medium. Also the number of grain diameters per stripe is higher for finer grains. Therefore, the coarse experimental setup deviates more from the idealized representation in the numerical model, in which sharp interfaces separate the stripes with different, uniform hydraulic conductivity.

Metrics of plume spreading and dilution, such as the second central moment and the flux-related dilution index, are calculated for the two cases (denoted as F3-3 and C3-3) shown in Fig. 4, as well as for analogous experiments performed in the same fine and coarse porous media but injecting the tracer from a neighboring injection port, denoted as F3-2 and C3-2, respectively. Figure 5 shows the second central moments computed from the concentration measurements and the simulation outputs at the outlet of the flow-through setup in the four different experiments. In both porous media, differences in plume spreading were observed when changing the plume injection port since the injected solute was transported along different streamlines. More important differences are observed between the two porous media; in fact, both the horizontal and vertical second central spatial moments indicate larger spreading in the coarse porous medium. The small difference of the second central moments between the experimental data and the model results shown in Fig. 5 indicates that the numerical model is able to predict the spreading of the steady-state plumes observed in the experiments.

Plume dilution was analyzed computing the flux-related dilution index [Eq. (8)] from the measured concentrations and flow rates in the flow-through experiments and from the outcomes of the transport simulations. The results for the four experiments are reported in Fig. 6. Not only the spreading but also the dilution of the plume is stronger in the heterogeneous anisotropic medium packed with coarse materials. This finding is consistent with the observations of peak concentrations illustrated in Fig. 4. Furthermore, the good agreement of the flux-related dilution index based on the experimental and the model results confirms the capability of the numerical

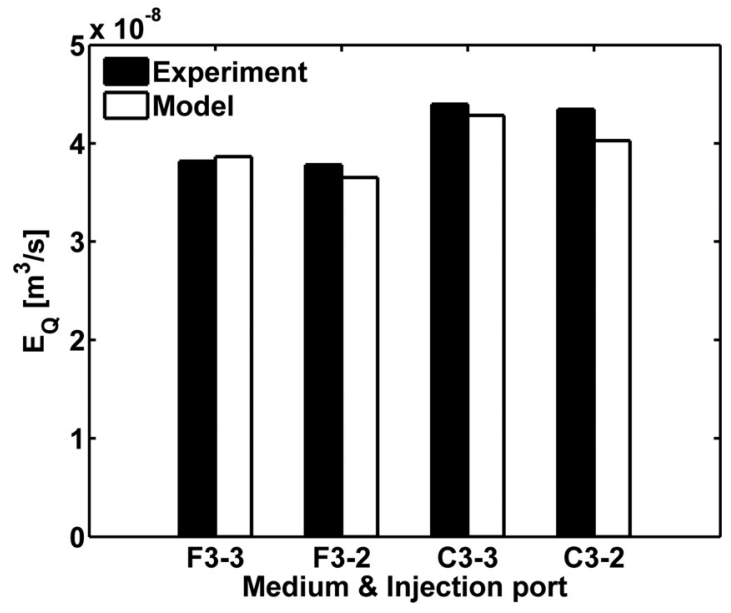

FIG. 6. Experimental and modeling results of the flux-related dilution index at the outlet of the flow-through system packed with fine (F) and coarse (C) materials using the central inlet port (3-3) and the neighboring one (3-2) as the tracer-injection ports.

model to accurately capture the flow and transport mechanisms controlling plume dilution.

\section{B. Reactive transport}

Mixing-controlled reactive transport was simulated for the four cases corresponding to the fine and coarse porous media and the injection conditions experimentally tested for conservative transport (Fig. 5). Three different critical mixing ratios, i.e., $0.8,0.5$, and 0.25 , representing different ratios of the inflow concentrations of the reactants $A$ and $B$ for identical stoichiometry of an instantaneous reaction, were selected for each reactive transport scenario. As an example, the red plume in Fig. 7 shows the development of the computed reactive plume with the critical mixing ratio of 0.5 in the heterogeneous anisotropic porous medium packed with fine materials (FF and FC grain sizes). In this case, we simulated that the central inlet port was used to inject the plume reactant $A$, whereas reactant $B$ was continuously injected from the

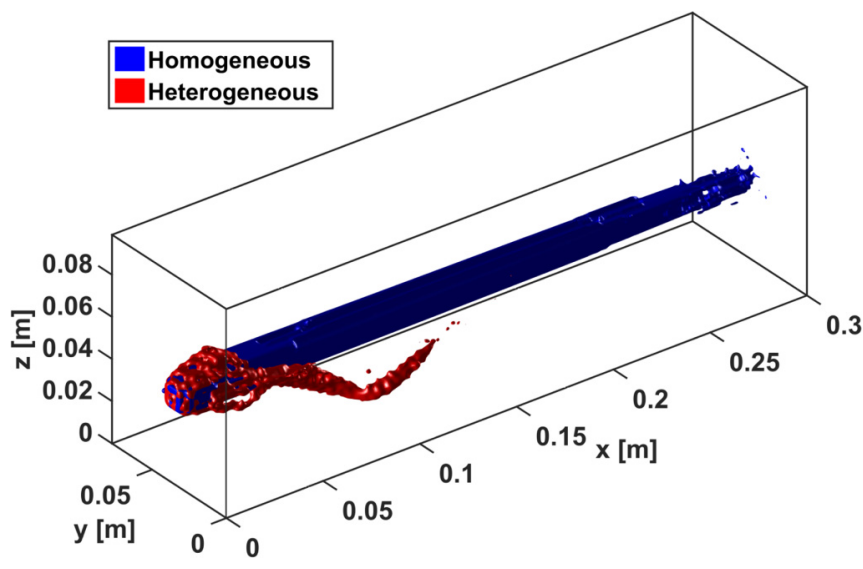

FIG. 7. Mixing-controlled reactive plumes $\left(X_{\text {crit }}=0.5\right)$ computed in a heterogeneous anisotropic porous medium and in a homogenous setup. Identical mass flux of reactant A was injected at the inlet in both setups. 
TABLE I. Metrics of reactive mixing (i.e., effective dispersion coefficient, plume length, and critical dilution index) computed for three values of $X_{\text {crit }}$ and for the four different heterogeneous anisotropic cases.

\begin{tabular}{|c|c|c|c|c|c|c|c|c|c|c|c|c|}
\hline \multicolumn{2}{|l|}{ Case } & \multicolumn{3}{|l|}{ F3-3 } & \multicolumn{2}{|l|}{ F3-2 } & \multicolumn{3}{|c|}{ C3-3 } & \multicolumn{3}{|c|}{$\mathrm{C} 3-2$} \\
\hline$X_{\text {crit }}[-]$ & 0.8 & 0.5 & 0.25 & 0.8 & 0.5 & 0.25 & 0.8 & 0.5 & 0.25 & 0.8 & 0.5 & 0.25 \\
\hline$D_{t, \mathrm{eff}} \times 10^{-8}\left[\mathrm{~m}^{2} \mathrm{~s}^{-1}\right]$ & 0.54 & 0.73 & 0.87 & 0.61 & 0.78 & 0.78 & 0.62 & 0.91 & 1.13 & 0.64 & 0.93 & 0.89 \\
\hline$L[\mathrm{~cm}]$ & 7.3 & 12.9 & 26.5 & 6.5 & 12.1 & 29.7 & 6.1 & 9.9 & 19.3 & 5.9 & 9.7 & 24.5 \\
\hline$L_{\mathrm{hom}}[\mathrm{cm}]$ & 14.5 & 34.4 & 83.7 & 14.5 & 34.4 & 83.7 & 14.4 & 34.0 & 82.7 & 14.4 & 34.0 & 82.7 \\
\hline $\mathrm{CDI} \times 10^{-7}\left[\mathrm{~m}^{3} \mathrm{~s}^{-1}\right]$ & 0.11 & 0.18 & 0.33 & 0.09 & 0.17 & 0.34 & 0.10 & 0.17 & 0.31 & 0.09 & 0.15 & 0.34 \\
\hline $\mathrm{CDI}_{\text {theor }} \times 10^{-7}\left[\mathrm{~m}^{3} \mathrm{~s}^{-1}\right]$ & 0.11 & 0.18 & 0.36 & 0.11 & 0.18 & 0.36 & 0.11 & 0.17 & 0.35 & 0.11 & 0.17 & 0.35 \\
\hline
\end{tabular}

surrounding inlet ports. The plume bends and spirals along the travel distance, and it disappears before reaching half the length of the domain. Figure 7 also shows a blue plume, which is the analogous reactive plume in a homogeneous porous medium. The simulation of the homogeneous porous medium was performed in a uniform hydraulic conductivity field ( $K=10^{-2} \mathrm{~m} / \mathrm{s}$, corresponding to a grain size of $1 \mathrm{~mm}$ ). Notice that, although the injected mass fluxes of the reactants in the two porous media are the same, the plumes differ in shape and length. In particular, in the homogeneous setup the reactive plume remains straight and reaches the outlet of the domain.

Table I summarizes the values of the selected metrics of reactive mixing computed (numerically or analytically) for the four cases of transport in heterogeneous anisotropic media and in the homogeneous domain. In all setups, the values of the reactive plume length vary considerably for different critical mixing ratios $\left(X_{\text {crit }}\right)$. The steady-state reactive plumes are longer for smaller critical mixing ratios, corresponding to the relative higher concentration of reactant $A$ at the inlet. In the range of considered $X_{\text {crit }}$, the plumes in the heterogeneous anisotropic media range from 5.9 to $29.7 \mathrm{~cm}$. For any given critical mixing ratio, the reactive plume length is shorter in the coarse porous medium (cases C3-3 and C3-2), consistently to the dilution observed for the conservative plumes (Fig. 6). Remarkable differences can be appreciated by comparing the results in the heterogeneous media with the plume length in the homogeneous domain. The latter is computed analytically according to Eq. (10). For all considered scenarios, the plume length in the heterogeneous anisotropic media is always less than half the length computed for the homogeneous case. Expressing the results as relative differences, the average reduction of the plume lengths in the heterogeneous cases is by factors of $1.25,2.11$, and 2.41 for the critical mixing ratios of $0.8,0.5$, and 0.25 , respectively. This clearly indicates a strong enhancement of mixing by the helical flow created in the heterogeneous anisotropic porous media. In fact, the complex flow fields with whirling streamlines induce significant deformation of the material surface of the plume causing an increased interfacial area and enhancing lateral mass exchange and, thus, the overall degradation rate.

The effective dispersion coefficients were computed according to Eq. (11) and the values of $D_{t, \text { eff }}$ are always larger than the local $D_{t}$ since the effects of heterogeneity and anisotropy are factored in the computation of the effective transverse dispersion coefficient. For instance, the local $D_{t}$ values at $3 \mathrm{~m} /$ day considering $X_{\text {crit }}=0.5$ are $0.16 \times 10^{-8} \mathrm{~m}^{2} / \mathrm{s}$, $0.41 \times 10^{-8} \mathrm{~m}^{2} / \mathrm{s}, 0.22 \times 10^{-8} \mathrm{~m}^{2} / \mathrm{s}$, and $0.54 \times 10^{-8} \mathrm{~m}^{2} / \mathrm{s}$ for the grain sizes FF, FC, CF, and $\mathrm{CC}$, respectively, and thus are smaller than the corresponding $D_{t, \text { eff }}$ reported in Table I. Similarly to the computed reactive plume lengths, the values of $D_{t, \text { eff }}$ show a more effective reaction enhancement in the heterogeneous media packed with coarser grains (C3-3 and C3-2).

The CDI is computed using the numerical simulations results, while its first-order approximation $\left(\mathrm{CDI}_{\text {theor }}\right)$ is computed by Eq. (12). The values of CDI and $\mathrm{CDI}_{\text {theor }}$ are also reported in Table I. For each scenario, the critical dilution index increases with decreasing mixing ratio, showing the same trend as the reactive plume length. However, for any given critical mixing ratio, the CDI values hardly vary for the four cases. These results show that the mixing required for the complete degradation of a plume reacting with a solute in the ambient water is unique and independent of the anisotropy and the heterogeneity of the porous media. The $\mathrm{CDI}_{\text {theo }}$ computed according to Eq. (12) shows a good approximation of the critical dilution index computed using the numerical model (mean normalized difference of $12 \%, 5 \%$, and $7 \%$ for the critical mixing ratio of $0.8,0.5$ and 0.25 , respectively).

\section{CONCLUSIONS}

In this study, we provided experimental observations of plume development in three-dimensional heterogeneous anisotropic porous media under helical flow conditions. Numerical simulations as well as metrics of transverse plume spreading, dilution, and reactive mixing were applied to interpret the experimental results. The outcomes of the investigation show the important effects of helical flow on plume dilution and reactive mixing in porous media. The link between complex three-dimensional flow features and transport processes in porous media, including the dynamics of plume deformation and mixing, is an area of broad interest for many disciplines but still largely unexplored. This might be caused by the difficulty in performing 3D experiments in complex flow-through setups and by the computational challenges in simulating transverse mixing in complex 3D flow fields. The current study contributes to overcoming these difficulties and may facilitate future investigations of solute transport in complex heterogeneous anisotropic porous media. The latter may evolve in different directions and concern both engineered (e.g., packed bed reactors and mixers) and natural systems (e.g., aquifers and oil reservoirs). For instance, the current understanding of contaminant plume evolution in aquifer systems will greatly benefit from high-resolution investigation taking into account three-dimensional features 
of flow and transport processes occurring in complex 3D sedimentary structures.

\section{ACKNOWLEDGMENTS}

This work was supported by the DFG (Deutsche Forschungsgemeinschaft, Grants No. RO 4169/3-1 and No. CI-26/11-1).

\section{APPENDIX A: LENGTH OF A REACTIVE PLUME IN A 3D HOMOGENEOUS ISOTROPIC POROUS MEDIUM}

In this Appendix, we derive the formula to compute the length of a steady-state reactive plume in a three-dimensional homogeneous isotropic porous medium. We consider a continuous injection from a square source and the analytical solution for the 3D steady-state distribution of a conservative tracer [Eq. (9)]:

$$
\begin{aligned}
X(x, y, z)= & \frac{1}{4}\left\{\operatorname{erf}\left[\frac{y+Y / 2}{2 \sqrt{D_{t} x / v}}\right]-\operatorname{erf}\left[\frac{y-Y / 2}{2 \sqrt{D_{t} x / v}}\right]\right\} \\
& \times\left\{\operatorname{erf}\left[\frac{z+Z / 2}{2 \sqrt{D_{t} x / v}}\right]-\operatorname{erf}\left[\frac{z-Z / 2}{2 \sqrt{D_{t} x / v}}\right]\right\} .
\end{aligned}
$$

At the end of the plume, the mixing ratio reached its critical value $X=X_{\text {crit }}$ at the central point (i.e., $y=z=0$ ) and Eq. (A1) simplifies to:

$$
X_{\text {crit }}=\left\{\operatorname{erf}\left[\frac{Y / 2}{2 \sqrt{D_{t} L / v}}\right]\right\}\left\{\operatorname{erf}\left[\frac{Z / 2}{2 \sqrt{D_{t} L / v}}\right]\right\} \text {. }
$$

Since the source of the plume is square $(Y=Z)$, Eq. (A2) can be further simplified to:

$$
X_{\text {crit }}=\left\{\operatorname{erf}\left[\frac{Y / 2}{2 \sqrt{D_{t} L / v}}\right]\right\}^{2}=\left\{\operatorname{erf}\left[\frac{Z / 2}{2 \sqrt{D_{t} L / v}}\right]\right\}^{2} .
$$

We can then solve for the length $L$ of the reactive plume:

$$
L=\frac{v Y^{2}}{16 D_{t}\left[\operatorname{erf}^{-1}\left(\sqrt{X_{\text {crit }}}\right)\right]^{2}} .
$$

\section{APPENDIX B: ANALYTICAL EXPRESSION OF THE CRITICAL DILUTION INDEX FOR A PLUME IN A 3D HOMOGENEOUS ISOTROPIC POROUS MEDIUM}

In the following, we derive an expression for the critical dilution index (CDI) in a three-dimensional homogeneous system. The CDI quantifies the mass flux dilution necessary to completely degrade a mixing-controlled reactive plume emitted from a continuous source and provides a direct link between conservative and reactive transport. For a square source $(Y=Z)$ in a three-dimensional system as considered in this study, inverse dynamic Péclet numbers in the transverse vertical and transverse horizontal directions can be defined as [50]:

$$
\varepsilon_{y}=\varepsilon_{z}=\frac{n x D_{t}}{q_{x} Y^{2}} .
$$

Considering the longitudinal distance at which the plume is completely degraded $(x=L)$ and inserting Eq. (B1) into Eq. (10), we obtain:

$$
\varepsilon_{y}=\varepsilon_{z}=\frac{1}{16\left[\operatorname{erf}^{-1}\left(\sqrt{X_{\text {crit }}}\right)\right]^{2}} .
$$

Taking the first-order approximation for the Taylor expression of the inverse error function, i.e., $\operatorname{erf}^{-1}\left(\sqrt{X_{\text {crit }}}\right) \approx$ $\frac{1}{2} \sqrt{\pi X_{\text {crit }}}$, Eq. (B2) becomes:

$$
\varepsilon_{y}=\varepsilon_{z}=\frac{1}{4 \pi X_{\text {crit }}} .
$$

The semianalytical expression of the flux-related dilution index for a conservative tracer in a 3D homogeneous setup reads as [50]:

$$
\begin{aligned}
& E_{Q}\left(\varepsilon_{y}, \varepsilon_{z}\right) \\
& =Y Z q_{x}\left(1-\sqrt{\frac{4 \pi \varepsilon_{y}}{4 \pi \varepsilon_{y}+1+f\left(\varepsilon_{y}\right)}}+\sqrt{4 \pi \exp (1) \varepsilon_{y}}\right) \\
& \quad \times\left(1-\sqrt{\frac{4 \pi \varepsilon_{z}}{4 \pi \varepsilon_{z}+1+f\left(\varepsilon_{z}\right)}}+\sqrt{4 \pi \exp (1) \varepsilon_{z}}\right) .
\end{aligned}
$$

For the common of sufficiently long plumes (i.e., $X_{\text {crit }}$ is small), the second term in both parentheses in Eq. (B4) tends to be 1 . Therefore, the terms in the parentheses are simplified to $\sqrt{4 \pi \exp (1) \varepsilon_{y}}$ and $\sqrt{4 \pi \exp (1) \varepsilon_{z}}$, respectively. By inserting the inverse dynamic Péclet numbers, calculated according to Eq. (B3), into Eq. (B4), we obtain:

$$
E_{Q}(L)=Y Z q_{x} \frac{\exp (1)}{X_{\text {crit }}} .
$$

Noting that $Y Z q_{x}$ is the flow rate through the source area and, thus, the flux-related dilution index at the inlet, the critical dilution index can be expressed as

$$
E_{Q}(L)=\frac{E_{Q}(0)}{X_{\text {crit }}} \exp (1) .
$$

[1] A. D. Stroock, S. K. W. Dertinger, A. Ajdari, I. Mezić, H. A. Stone, and G. M. Whitesides, Science 295, 647 (2002).

[2] V. Hessel, H. Löwe, and F. Schönfeld, Chem. Eng. Sci. 60, 2479 (2005).

[3] J. B. Weiss and A. Provenzale, Transport and Mixing in Geophysical Flows (Springer, Berlin/Heidelberg, 2008).
[4] B. Jha, L. Cueto-Felgueroso, and R. Juanes, Phys. Rev. E 84, 066312 (2011).

[5] J. M. Ottino, The Kinematics of Mixing (Cambridge University, Cambridge, 1989).

[6] B. Bijeljic, A. Raeini, P. Mostaghimi, and M. J. Blunt, Phys. Rev. E 87, 013011 (2013). 
[7] A. Paster, T. Aquino, and D. Bolster, Phys. Rev. E 92, 012922 (2015).

[8] C. Simonnet and A. Groisman, Phys. Rev. Lett. 94, 134501 (2005).

[9] T. W. Willingham, J. C. Werth, and A. J. Valocchi, Environ. Sci. Technol. 42, 3185 (2008).

[10] M. Norouzi Rad, N. Shokri, and M. Sahimi, Phys. Rev. E 88, 032404 (2013).

[11] P. de Anna, J. Jimenez-Martinez, H. Tabuteau, R. Turuban, T. Le Borgne, M. Derrien, and Y. Meheust, Environ. Sci. Technol. 48, 508 (2014).

[12] A. M. Tartakovsky, G. D. Tartakovsky, and T. D. Scheibe, Adv. Water Resour. 32, 1674 (2009).

[13] A. M. Tartakovsky, Phys. Rev. E 82, 026302 (2010).

[14] D. L. Hochstetler, M. Rolle, G. Chiogna, C. M. Haberer, P. Grathwohl, and P. K. Kitanidis, Adv. Water Resour. 54, 1 (2013).

[15] M. Icardi, G. Boccardo, D. L. Marchisio, T. Tosco, and R. Sethi, Phys. Rev. E 90, 013032 (2014).

[16] D. R. Lester, G. Metcalfe, and M. G. Trefry, Phys. Rev. E 90, 063012 (2014).

[17] M. Rolle and P. Kitanidis, Adv. Water Resour. 71, 186 (2014).

[18] M. Rolle, G. Chiogna, D. L. Hochstetler, and P. K. Kitanidis, J. Contam. Hydrol. 153, 51 (2013).

[19] Y. Edery, I. Dror, H. Scher, and B. Berkowitz, Phys. Rev. E 91, 052130 (2015).

[20] O. A. Cirpka, E. O. Frind, and R. Helmig, J. Contam. Hydrol. 40, 159 (1999).

[21] M. De Simoni, J. Carrera, X. Sánchez-Vila, and A. Guadagnini, Water Resour. Res. 41, W11410 (2005).

[22] D. Bolster, D. A. Benson, T. Le Borgne, and M. Dentz, Phys. Rev. E 82, 021119 (2010).

[23] T. Le Borgne, M. Dentz, P. Davy, D. Bolster, J. Carrera, J. de Dreuzy, and O. Bour, Phys. Rev. E 84, 015301 (2011).

[24] G. Chiogna, D. L. Hochstetler, A. Bellin, P. K. Kitanidis, and M. Rolle, Geophys. Res. Lett. 39, L20405 (2012).

[25] A. Paster, D. Bolster, and D. A. Benson, Water Resour. Res. 49, 1 (2013).

[26] M. Bakker and K. Hemker, Adv. Water Resour. 27, 1075 (2004).

[27] K. Hemker, E. van den Berg, and M. Bakker, Ground Water 42, 234 (2004).
[28] F. Stauffer, Adv. Water Resour. 30, 2194 (2007).

[29] G. Chiogna, M. Rolle, A. Bellin, and O. A. Cirpka, Adv. Water Resour. 73, 134 (2014).

[30] G. Chiogna, O. A. Cirpka, M. Rolle, and A. Bellin, Water Resour. Res. 51, 261 (2015).

[31] D. R. Lester, M. Dentz, T. Le Borgne, and F. P. J. de Barros (unpublished).

[32] O. A. Cirpka, G. Chiogna, M. Rolle, and A. Bellin, Water Resour. Res. 51, 241 (2015).

[33] Y. Ye, G. Chiogna, O. A. Cirpka, P. Grathwohl, and M. Rolle, Phys. Rev. Lett. 115, 194502 (2015).

[34] G. Nichols, Sedimentology and Stratigraphy (Wiley-Blackwell, Chichester, 2009).

[35] A. Hazen, Annu. Rep. State Board of Health Mass. 24, 541 (1892).

[36] D. W. Pollock, Ground Water 26, 743 (1988).

[37] O. A. Cirpka, F. P. J. de Barros, G. Chiogna, M. Rolle, and W. Nowak, Water Resour. Res. 47, W06515 (2011).

[38] G. Chiogna, C. Eberhardt, P. Grathwohl, O. A. Cirpka, and M. Rolle, Environ. Sci. Technol. 44, 688 (2010).

[39] I. Klenk and P. Grathwohl, J. Contam. Hydrol. 58, 111 (2002).

[40] M. Rolle, D. L. Hochstetler, G. Chiogna, P. K. Kitanidis, and P. Grathwohl, Transp. Porous Media 93, 347 (2012).

[41] U. M. Scheven, Phys. Rev. Lett. 110, 214504 (2013).

[42] Y. Ye, G. Chiogna, O. A. Cirpka, P. Grathwohl, and M. Rolle, Water Resour. Res. 51, 5582 (2015).

[43] M. Rolle, C. Eberhardt, G. Chiogna, O. A. Cirpka, and P. Grathwohl, J. Contam. Hydrol. 110, 130 (2009).

[44] P. K. Kitanidis, Water Resour. Res. 30, 2011 (1994).

[45] O. A. Cirpka, A. Olsson, Q. Ju, M. A. Rahman, and P. Grathwohl, Ground Water 44, 212 (2006).

[46] O. A. Cirpka and A. J. Valocchi, Adv. Water Resour. 30, 1668 (2007).

[47] P. A. Domenico and V. V. Palciauskas, Ground Water 20, 303 (1982).

[48] G. Chiogna, O. A. Cirpka, P. Grathwohl, and M. Rolle, Water Resour. Res. 47, W02505 (2011).

[49] P. Fornasini, The Uncertainty in Physical Measurements (Springer-Verlag, New York, 2008).

[50] Y. Ye, G. Chiogna, O. A. Cirpka, P. Grathwohl, and M. Rolle, J. Contam. Hydrol. 172, 33 (2015). 